\title{
Polymer matrix ferroelectric composites under pressure: Negative electric capacitance and glassy dynamics
}

\author{
Szymon Starzonek ${ }^{1, a}$, Aleksandra Drozd-Rzoska ${ }^{1}$, Sylwester J. Rzoska ${ }^{1}$, Kena Zhang ${ }^{2}$, Emilia Pawlikowska ${ }^{1}$, \\ Aleksandra Kȩdzierska-Sar ${ }^{1}$, Mikolaj Szafran ${ }^{3}$, and Feng Gao ${ }^{2}$ \\ 1 Institute of High Pressure Physics of the Polish Academy of Sciences, ul. Sokołowska 29/37, 01-142 Warsaw, Poland \\ 2 State Key Laboratory of Solidification Processing, MIIT Key Laboratory of Radiation Detection Materials and Devices, \\ NPU-QMUL Joint Research Institute of Advanced Materials and Structure, School of Material Science and Engineering, \\ Northwestern Polytechnical University, Xi'an, 710072, China \\ 3 Faculty of Chemistry, Warsaw University of Technology, ul. Noakowskiego, 00-664 Warsaw, Poland
}

Received 5 January 2019 and Received in final form 5 July 2019

Published online: 9 September 2019

(c) The Author(s) 2019. This article is published with open access at Springerlink.com

\begin{abstract}
This report presents the results of high-pressure and broadband dielectric spectroscopy studies in polyvinylidene difluoride (PVDF) and barium strontium titanate (BST) microparticles composites (BST/PVDF). It shows that the Arrhenius behaviour for the temperature-related dynamics under atmospheric pressure is coupled to Super-Arrhenius/Super-Barus isothermal pressure changes of the primary relaxation time. Following these results, an explanation of the unique behaviour of the BST/PVDF composite is proposed. Subsequently, it is shown that when approaching the GPa domain the negative electric capacitance phenomenon occurs.
\end{abstract}

\section{Introduction}

Glass transition constitutes one of the key properties for polymers processing and their fundamental characterisation [1-3]. It can occur for polymeric melts or in the solid state, where the continuous solidification of segmental motions occurs. Previtreous changes of dynamic properties can start even $200 \mathrm{~K}$ above the glass temperature $\left(T_{g}\right)$ and they are related to the gradual slowing down, from even $10^{-14} \mathrm{~s}$ to $\tau\left(T_{g}\right)=10^{2} \mathrm{~s}$ [1]. All these show the far remote impact of the glass transition also on the primary relaxation time, viscosity, diffusion, conductivity $[1,4]$. The broadband dielectric spectroscopy (BDS) can be recognized as the key research method for monitoring the previtreous behaviour due to its unique possibilities of getting insight into even 15 decades in frequency/time, both for orientational and translational properties $[5,6]$. A new kind of polymer-based composite materials can be obtained when dispersing solid nanoor micro-particles in the polymer matrix, what can yield substantially changed/improved mechanical, optical, and dielectric properties. Such favourite features have led to a vast range of applications, ranging from commodity polymer materials, to advanced materials in aerospace, medical, and electronics industries. New features of polymer-

\footnotetext{
${ }^{a}$ e-mail: starzoneks@unipress.waw.pl (corresponding author)
}

solid particles composites appear due to the beneficial combination of components properties and added value features appearing as the consequence of the guest-host (solid particles-polymer matrix) interactions [7-9]. One can expect that they can be particularly efficient when linking a polymer showing ferroelectric features with ferroelectric solid nano- or micro-particles. This is the case of polyvinylidene difluoride (PVDF) and barium strontium titanate (BST) particles. PVDF exhibits the ferroelectricparaelectric phase transition with the Curie temperature located near melting at $T_{m}=438-473 \mathrm{~K}$ [10-12]. On cooling towards the glass temperature $\left(T_{g} \sim 235 \mathrm{~K}\right)$ the SuperArrhenius (SA) changes of the primary relaxation time, associated with the apparent and temperature dependence activation energy, take place [13-15]. Worth mentioning is the importance of PVDF for fundamental model studies and also its enormous practical significance: associated not only with ferroelectricity but also with mechanical properties, stability resistance to solvents, acids and bases. Consequently, PVDF is applied in advanced electronic devices, in the chemical industry, modern medical equipment and in the food industry. For the latter, its non-toxicity PVDF is particularly important $[2,3]$. Barium strontium titanate belongs to the group of classical ferroelectric solids with the perovskite crystalline structure [16-18]. The applied in studies presented below BST powder had grains with an average diameter $d=0.7 \mu \mathrm{m}$ and a Curie temperature $T_{C}=286 \mathrm{~K}$ [19]. BST is broadly applied in modern 
electronic, particularly within integrated circuits. Its importance for innovative tuneable devices up to even $\mathrm{THz}$ range is also known $[17,18]$.

It was recently shown that for BST/PVDF (10\% vol. fraction of BST) the dynamics between $T_{m}$ and $T_{g}$ is clearly Arrhenius (A) type, with a constant activation energy. On cooling below $T_{g}$, a new SA relaxation process associated with the relaxor glassy nature of BST appears $[19,20]$. Notable is the coincidence of $T_{g}$ in PVDF and the $\mathrm{A} \rightarrow \mathrm{SA}$ crossover temperature in the $\mathrm{BST} / \mathrm{PVDF}$ composite [19].

This report presents the results of high-pressure BDS studies in a BST/PVDF composite showing that the Arrhenius dynamics for the temperature evolution under atmospheric pressure is coupled to Super-Arrhenius/SuperBarus isothermal pressure changes. Following this finding, an explanation of the unique dynamics in the $\mathrm{BST} / \mathrm{PVDF}$ composite is proposed. When approaching the GPa domain an unusual negative electric capacitance phenomenon [21], in the bulk composite sample, can appear.

\section{Experimental}

Broadband dielectric spectroscopy studies were carried out using BDS Novocontrol spectrometer operating from $0.1 \mathrm{~Hz}$ to $3 \mathrm{GHz}$ in temperature studies under atmospheric pressure. The temperature control was reached due to the Quattro Novocontrol system. High-pressure studies, for $f<10 \mathrm{MHz}$, were carried out via the Alpha impedance analyser linked to the high-pressure system designed and built in IHPP PAS. The system enables the computercontrolled changes of pressure and temperature. It is notable that BDS measurements in situ under pressure for higher frequencies are still the unsolved implementation problem.

The $\mathrm{Ba}_{0.6} \mathrm{Sr}_{0.4} \mathrm{TiO}_{3}$ (BST) powders were fabricated by the solid-state reaction method, using materials of $\mathrm{BaCO}_{3}$, $\mathrm{SrCO}_{3}$ and $\mathrm{TiO}_{2}$ mixed in stoichiometric composition, heated at $1673 \mathrm{~K}$ and the subsequent grinding for $2 \mathrm{~h}$. The surface of BST particles was modified by silane coupling agent KH550 to enhance the connectivity between the BST fillers and the PVDF matrix. PVDF and BST powders were mixed in a DMF solvent. The mixture was ultrasonic dispersed for $2 \mathrm{~h}$ and stirring dispersed for $6 \mathrm{~h}$, subsequently tape-casted on a glass substrate to obtain the thick film. After that, the film was kept in a vacuum oven overnight at $433 \mathrm{~K}$ to evaporate the organic solvent of DMF. Then the BST/PVDF films with $0.1 \sim 0.2 \mathrm{~mm}$ in thickness were produced by hot pressed at $393 \mathrm{~K}$ and $100 \mathrm{MPa}$ for $2 \mathrm{~h}$. The average particle diameter of BST particles was $0.6 \mu \mathrm{m}$, as measured by the Laser Diffraction Particle Size Analyzer.

\section{Results and discussion}

Results presented below are for BST/PVDF composites, with a BST amount ranging between $10 \%$ to $50 \%$ vol. fraction of BST. Examples of BDS spectra under $P=0.5 \mathrm{GPa}$

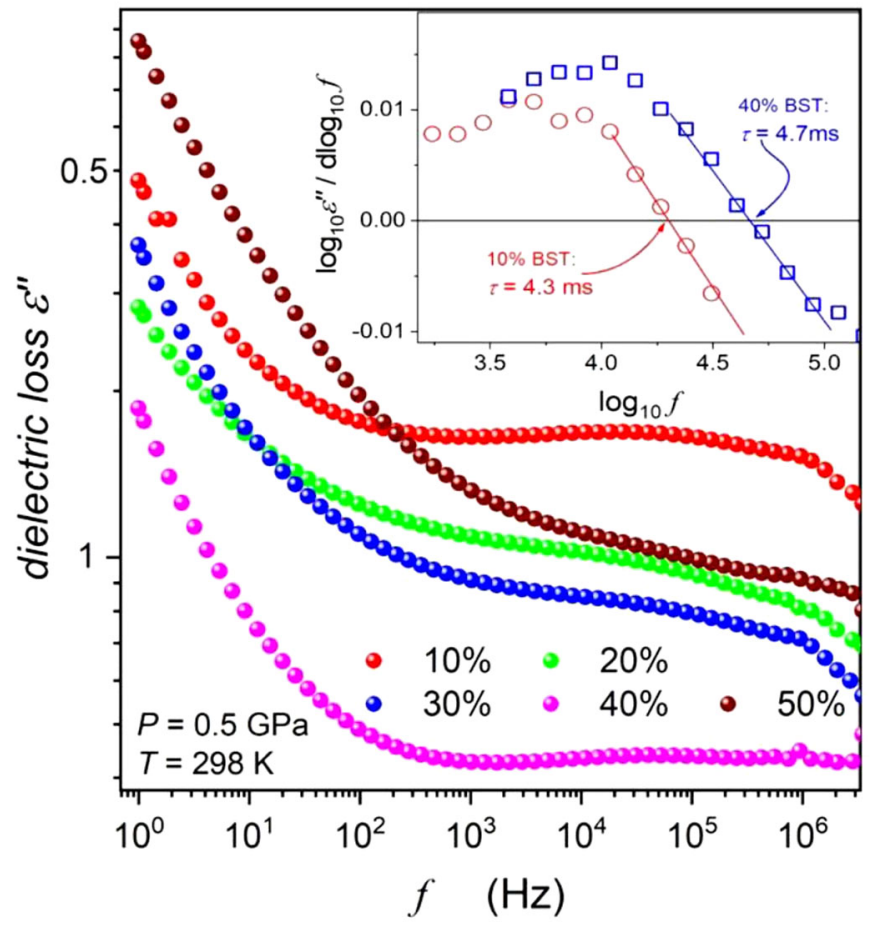

Fig. 1. The imaginary part of dielectric permittivity in BST/PVDF composites with different volume fractions of $\mathrm{BST}$, for $T=293 \mathrm{~K}$ and $P=0.5 \mathrm{GPa}$. The inset shows results of the derivative analysis focusing on the detection of the peak frequency and then the primary relaxation time.

are shown in fig. 1 . Notable is the suppression of the primary relaxation process when increasing the amount of BST - it ceases to be detectable for $v=50 \%$.

To test the possible influence of pressure and BST amount on the peak frequency of the loss curves and then the primary relaxation time the distortion-sensitive analysis presented in the inset in fig. 1 was carried out. It shows $\sim 10 \%$ increase (slowing-down) of the primary relaxation time when rising the amount of BST from $10 \%$ to $40 \%$. For the highest amount of BST (50\%) the manifestation of the primary loss curve ceased to be detectable. The relaxation time is determined by the peak frequency of the loss curve as $\tau=1 / 2 \pi f_{\text {peak }}$ or alternatively from the condition $\mathrm{d} \log \varepsilon^{\prime \prime}\left(f=f_{\text {peak }}\right) / \mathrm{d} \log f=0$.

The manifestation of the primary relaxation process under atmospheric pressure is qualitatively different: the amount of BST has a negligible influence on the "strength" of the primary loss curve and there is no impact on the primary relaxation time, as shown in fig. 2 .

It employs the alternative to fig. $1\left(\varepsilon^{\prime \prime}(f), T=\right.$ const $)$ way of presentation of dielectric permittivity data: $\varepsilon^{\prime \prime}(T)$, $f=$ const, popular in research societies focusing on ferroelectrics and polymers. We would like to indicate the omitted so far simple and "universal" pattern of behaviour associated with such representation. As shown by solid lines in fig. 2: $\log \varepsilon^{\prime \prime}(T)=\ln \varepsilon^{\prime \prime} / \ln 10=c_{ \pm}+b_{ \pm} T$ and then $\varepsilon^{\prime \prime}(T)=C_{ \pm} \exp \left(B_{ \pm} T\right)$, where $C=\exp \left(\ln 10 c_{ \pm}\right)$, $B=\ln 10 b_{ \pm}$and " \pm " are for $T>T_{\text {peak }}$ and $T<T_{\text {peak }}$, respectively. 


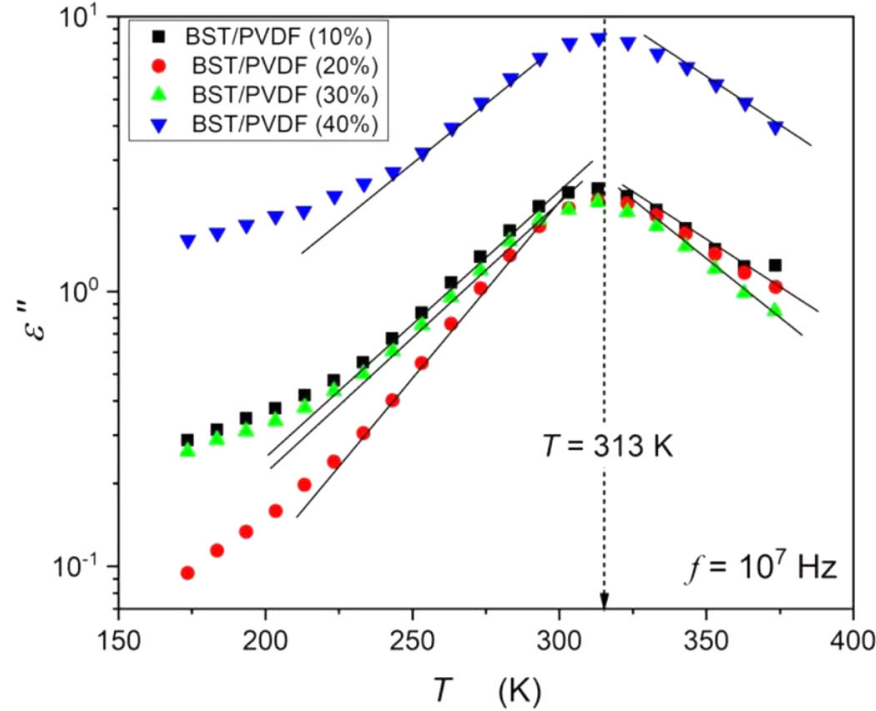

Fig. 2. The temperature evolution of the imaginary part of dielectric permittivity $(P=0.1 \mathrm{MPa})$ for the constant frequency $f=1 \mathrm{MHz}$, in BST/PVDF composites with different amounts of BST. The arrow shows the "peak temperature" coupled to the relaxation time $\tau=1 / 2 \pi f=0.16 \mu$ s.

When discussing the previtreous evolution of the primary relaxation time one can recall the general dependence for describing their temperature and pressure evolutions [22]:

$$
\begin{aligned}
\tau(T, P) & =\tau_{0} \exp \left(\frac{E_{a}(T)+P V_{a}(P)}{R T}\right) \\
& =\left[\tau_{0} \exp \left(\frac{E_{a}(T)}{R T}\right)\right] \exp \left(\frac{P V_{a}(P)}{R T}\right),
\end{aligned}
$$

where the apparent activation energy $E_{a}(T)$ and the apparent activation volume $V_{a}(P)$ have been introduced.

Regarding the pre-factor $\tau_{0}$, for the basic Arrhenius dependence (see below) theoretically the "universal" terminal value $\tau_{0}=k_{B} T / h \sim 10^{-14} \mathrm{~s}$ reached at very high temperatures is indicated [23]. However, in practice for various systems ranges $\tau_{0}=10^{-10} \mathrm{~s}$ and $10^{-16} \mathrm{~s}[24-27]$.

For isobaric studies, under constant reference pressure $P_{\text {ref }}$ the above equation takes the form

$$
\begin{aligned}
\tau(T) & =\left[\tau_{0} \exp \left(\frac{\left(P V_{a}\right)_{r e f}}{R T}\right)\right]_{r e f} \exp \left(\frac{E_{a}(T)}{R T}\right), \\
P & =\text { const }
\end{aligned}
$$

where the term in square bracket is the reference prefactor defined by the onset temperature and pressure under which studies are carried out: its values can range between $\tau_{\text {ref }} \approx 10^{-10} \mathrm{~s}$ and $\tau_{\text {ref }} \approx 10^{2} \mathrm{~s}$, depending on the selected isobar.

For studies under atmospheric pressure, where one can assume $P \sim 0$, eq. (2) is approximated by the SuperArrhenius (SA) relation [1]

$$
\tau(T)=\tau_{0} \exp \left(\frac{E_{a}(T)}{R T}\right) .
$$

For the constant value of the activation energy $\left(E_{a}(T)=E_{a}=\right.$ const $)$ in the given temperature domain one obtains the basic Arrhenius (A) dependence, yielding a linear dependence for $\log \tau(T)$ vs. $1 / T$ plot. For the isothermal case, pressure-related approaching the glass transition point one obtains:

$$
\begin{aligned}
\tau(P) & =[\tau(T)]_{r e f} \exp \left(\frac{P V_{a}(P)}{R T}\right), \\
T & =\text { const }
\end{aligned}
$$

where the term in the square bracket is the reference prefactor defined by the onset pressure and temperature of the selected isotherm: its values can range between $\tau_{\text {ref }} \approx$ $10^{-10} \mathrm{~s}$ and $\tau_{\text {ref }} \approx 10^{2} \mathrm{~s}$, depending on the isotherm.

Equation (4) recalls the empirical dependence proposed by Barus $\tau(P) \propto \exp (A P)$ [28]. If for the given pressure range $V_{a}(P)=V_{a}=$ const, it yields the linear behaviour for $\log \tau(P)$ vs. $P$ plot. Taking into account that eq. (3) with the apparent temperature-dependent activation energy is known as the Super-Arrhenius (SA) relation, one can name eq. (4) as the Super-Barus (SB) dependence. The application of eqs. (1)-(4) for portraying SA or SB behaviour requires the prior knowledge of $E_{a}(T)$ and $V_{a}(P)$ functional forms. This constitutes the unsolved problem and "ersatz" dependences have to be used. For SA behaviour temperature studies, the Vogel-Fulcher-Tammann (VFT) relation is applied [1,29-32]:

$$
\begin{aligned}
\tau(T) & =\tau_{0} \exp \left(\frac{D_{T} T_{0}}{T-T_{0}}\right), \\
P & =\text { const },
\end{aligned}
$$

where $T_{0}<T_{g}$ is the singular VFT temperature and $D_{T}$ is the fragility strength coefficient. Equation (5) leads to the activation energy $E_{a}(T)=\left(R D_{T} T_{0}\right) T /\left(T-T_{0}\right)$, when comparing relations (3) and (5).

It is notable that the VFT equation is so often and effectively applied that it is indicated amongst universal patterns for previtreous effects on approaching the glass temperature. As replacement relation for the SB eq. (4) the following relation was proposed [33]:

$$
\begin{aligned}
\tau(P) & =\tau_{\text {ref }} \exp \left(\frac{D_{P} P_{0}}{P_{0}-P}\right), \\
T & =\text { const }
\end{aligned}
$$

where $P_{0}>P_{g}$ is the singular pressure and $D_{P}$ is the fragility strength coefficient for the pressure path, that yields $V_{a}(P)=R T D_{P} /\left(P_{0}-P\right)$.

It can be reduced to the basic Barus relation [28] for large values of $D_{P}$ and $P_{0}$, particularly if experimental data terminates well below $P_{0}$. One should recall that already in 1972 the following relation was empirically proposed by Whalley and Johari [34] for portraying viscosity changes in glycerol and in ref. [35] for the primary relaxation time in few "fragile" glass-formers:

$$
\tau(P)=\frac{A}{P_{0}-P} .
$$


However, it is not compatible with the basic Barus equation [28] and the SB eq. (6). It also omits the link to the fragility. The latter is considered as one of the key concepts of "universality" in the glass transition physics. To measure the degree of the SA behaviour the fragility coefficient was introduced $[32,36]: m_{T}=$ $\mathrm{d} \log \tau(T) / \mathrm{d}\left(T_{g} / T\right)$ for $T=T_{g}$. For its pressure parallel $m_{P}=\mathrm{d} \log \tau(P) / \mathrm{d}\left(P / P_{g}\right)$ is proposed. The basic way of determining fragilities is the application of the "Angell plot" $\log \tau(T)$ vs. $\left(T_{g} / T\right)[32,36]$ or its pressure counterpart $\log \tau(T)$ vs. $\left(P / P_{g}\right)[37,38]$. Linking the definition of fragilities and eqs. $(5),(6)$ one obtains

$$
\begin{aligned}
m_{T}(T) & =\frac{D_{T}\left(T_{0} / T_{g}\right)}{\left(1-T_{0} / T_{g}\right)^{2}} \\
\text { and } m & =m_{T}(T)=D_{T} \frac{r_{T}}{\left(1-r_{T}\right)^{2}}, \\
m_{P}(P) & =\frac{D_{P}\left(P_{0} / P_{g}\right)}{\left(P_{0} / P_{g}-1\right)^{2}} \\
\text { and } m & =m_{P}(P)=D_{P} \frac{r_{P}}{\left(r_{P}-1\right)^{2}},
\end{aligned}
$$

where coefficients $r_{T}=T_{0} / T_{g}$ and $r_{P}=P_{0} / P_{g}, m_{T}(T)$ and $m_{P}(P)$ are steepness indexes for $T>T_{g}$ and $P<P_{g}$. It is assumed that $\tau\left(T_{g}, P_{g}\right)=100 \mathrm{~s}$.

High-pressure studies often terminate at some values of pressure determined by experimental possibilities, often well below the glass transition $\tau\left(T_{g}, P_{g}\right)=100 \mathrm{~s}$. This is particularly important in high-pressure BDS studies for which relatively large pressurised volume $\left(V \sim 5 \mathrm{~cm}^{3}\right)$ can be advised for high-resolution and non-biased measurements requiring a proper design of the measurement capacitor (see, for instance, ref. [39]). The reliable portrayals of $\tau(T)$ and/or $\tau(P)$ experimental data even well below $\left(T_{g}, P_{g}\right)$ enables estimations of $\left(T_{g}, T_{0}, D_{T}\right)$ and $\left(P_{g}, P_{0}, D_{P}\right)$. Then applying eqs. (8) and (9) one can calculate $m$ and $m_{P}$ fragilities.

Figure 3 presents the pressure evolution of the primary relaxation time for two amounts of BST in BST/PVDF composites, at the room temperature. The obtained parameters show the notable $\mathrm{SB}$ dynamics with $P_{g}=$ $700 \mathrm{MPa}, P_{0}=0.96 \mathrm{GPa}, D_{P}=4.8$ and then $m_{P}=20$ for the BST/PVDF (10\%) composite and $P_{g}=700 \mathrm{MPa}$, $P_{0}=1.3 \mathrm{GPa}, D_{P}=6.2$ and $m_{P}=40$ for the BST $/ \mathrm{PVDF}$ (40\%) composite. The increase of the amount of BST microparticles in the BST/PVDF composite strongly increases the high-pressure-related fragility.

The temperature behaviour of the primary relaxation time, for BST/PVDF composites with $10 \%$ and $40 \%$ of $\mathrm{BST}$, is presented in fig. 4. It shows the following key "unique" features of dynamics in a PVDF/BST composite:

i) the clear SA dynamics (changeable activation energy) in PVDF transforms to the Arrhenius pattern (constant activation energy) in a BST/PVDF composite, for $T>T_{g}$

ii) for $T<T_{g}$ the BST-based $\mathrm{SA}$ relaxation process emerges in the composite: the question arises why it was absent for $T>T_{g}$;

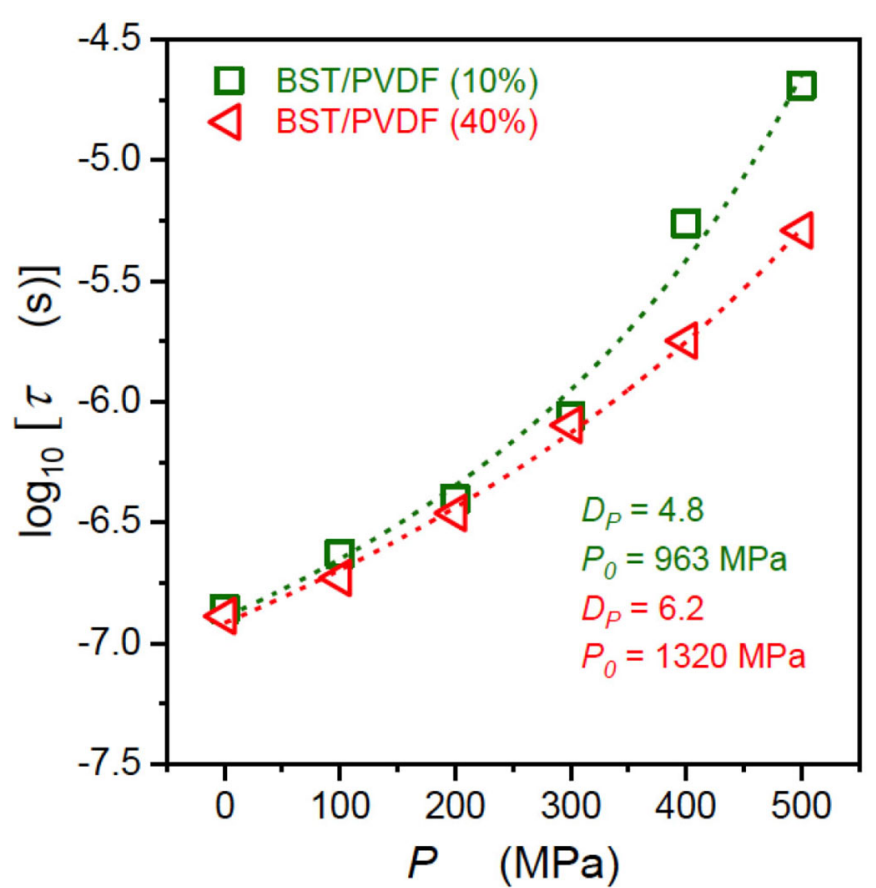

Fig. 3. The pressure evolution of the primary relaxation time in BST/PVDF composites with different amounts of BST microparticles: $T=270 \mathrm{~K}$.

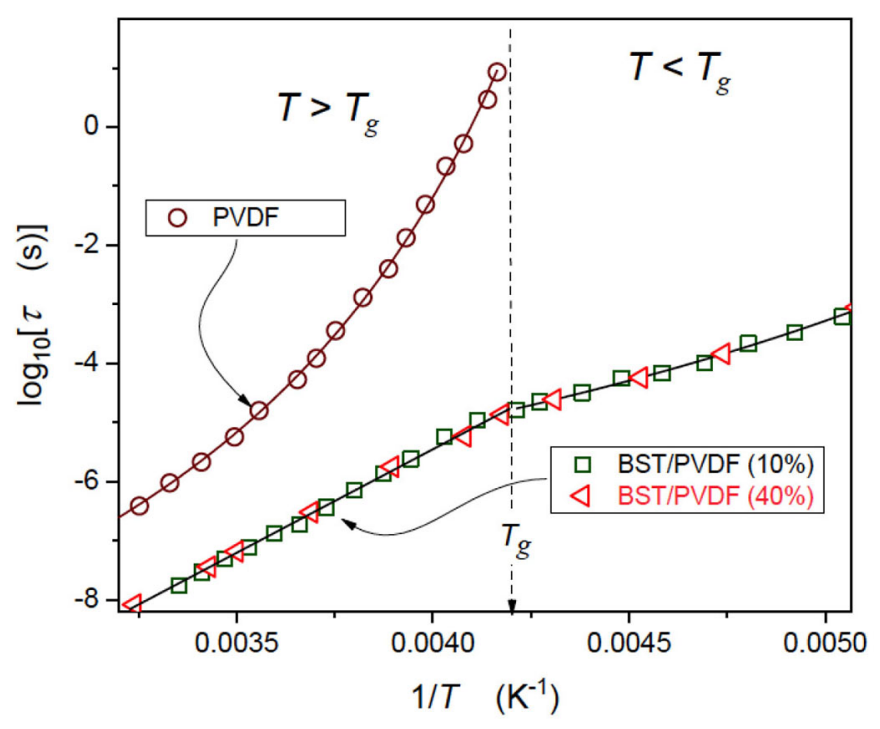

Fig. 4. The temperature evolution of the primary relaxation time in PVDF and BST/PVDF composites.

iii) the "dynamic" crossover $\mathrm{A} \rightarrow \mathrm{SA}$ in the BST/PVDF composite occurs at the PVDF glass temperature;

iv) for the pressure path, the dynamics follow the SB one, despite the "Arrhenius background" for the temperature path;

v) for the pressure path the change of the amount of BST in the composite influences the fragility and the value of the primary relaxation time. There is no such impact for the temperature path under atmospheric pressure. 

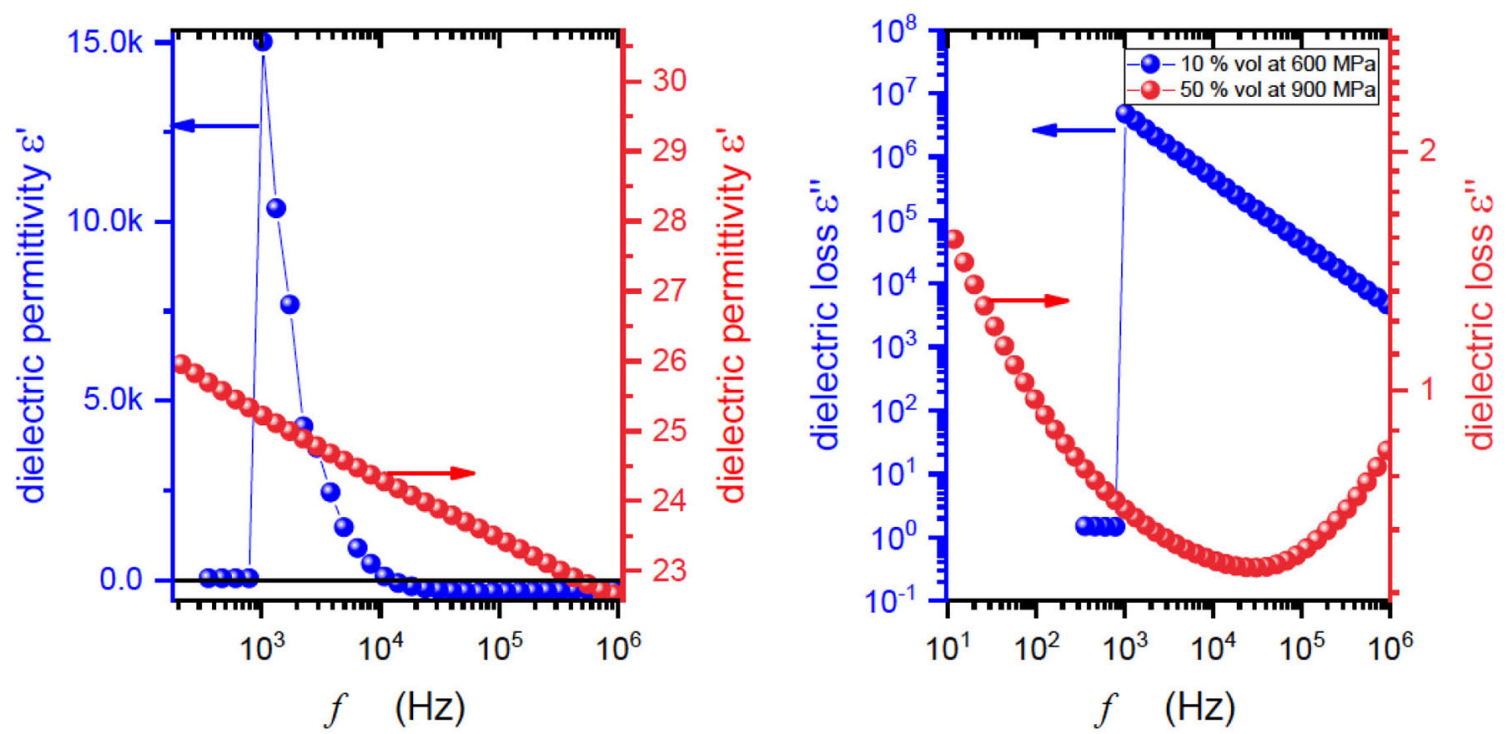

Fig. 5. Dielectric spectra for the sample BST/PVDF composites with $10 \%$ and $50 \%$ vol. fraction of BST at $T=298 \mathrm{~K}$.

The increase of pressure above $0.5 \mathrm{GPa}$ dramatically changes the dielectric spectrum for the BST/PVDF (10\%) composite, as shown in fig. 5. The real part of dielectric permittivity is slightly negative $\varepsilon^{\prime}<0$ for $f<20 \mathrm{kHz}$. On further decreasing the frequency it enormously rises to $\varepsilon^{\prime} \approx 15000$ and for $f=9.1 \mathrm{kHz}$ it collapses to $\varepsilon^{\prime}=0$. For the imaginary, loss-related, part of dielectric permittivity there is a strong increase strictly following the pattern $\varepsilon^{\prime \prime}(f) \propto f^{-k}$, in the whole temperature range up to $f=9.1 \mathrm{kHz}$, where it sharply decreases to the constant value $\varepsilon^{\prime \prime}(f) \propto 2$. For BST/PVDF (50\%) an atypical dielectric spectrum appears already when approaching $P=0.9 \mathrm{GPa}$. The real part of dielectric permittivity $\varepsilon^{\prime}(f)$ almost linearly increases, from 22.5 to 27 . The behaviour of $\varepsilon^{\prime}(f)$ has a parabolic form with the minimum at $f=30 \mathrm{kHz}$. All these shows the importance of a proper ration of PVDF and BST for the appearance of metamaterial dielectric features in BST/PVDF composites.

When discussing the spectra presented in fig. 4 one should recall the basic definition linking real and imaginary parts of dielectric permittivity to the electric capacitance $C$ and the resistivity $R: \varepsilon^{\prime}=C / C_{0}$ and $\varepsilon^{\prime \prime}=$ $1 / \omega R C_{0}$, where $C_{0}$ is the capacitance of the empty measurement capacitor and $\omega=2 \pi f[5]$.

Hence, the behaviour presented in fig. 5 can be linked to the phenomenon of negative electric capacitance. It is recognized as "the New Hope" for low energetic and ultrafast transistors and integrated circuits in the XXI Century. This phenomenon has a long history, but the experimental evidence remained puzzling until recently [21,40-45]. We would like to stress that the picture presented in fig. 5 is for the bulk sample and $d \sim 1 \mathrm{~mm}$ thick sample.

\section{Conclusions}

The results presented above focus on the BDS-related high-pressure behaviour of BST/PVDF microcomposites. First, the ways of the consistent portrayal of the pressure and temperature evolutions of the primary relaxation time, or related dynamic properties, in glassy systems were concluded in eqs. (1)-(7). Subsequently, the unique behaviour of dynamics in BST/PVDF composites resulted from pressure and temperature studies of the primary relaxation process was presented and summarized in points i) $-v)$. In the opinion of the authors, this can be explained assuming that the electric field from ferroelectric $\mathrm{BaTiO}_{3}$ microinclusions can order and restrict segmental motions to the relaxation process, with the same activation energy for all segments. On the other hand, the electric field from the ferroelectric PVDF matrix acts on domains within $\mathrm{BaTiO}_{3}$ particles, blocking their freedom and the development of the relaxor-type relaxation process above $T_{g}$. The question arises if some feedback process between PVDF and BST particles increasing interactions appeared. When reaching $T_{g}$ on cooling the amorphous solidification of PVDF has to strengthen interactions within polymer what can break the PVDF-BST interaction and the relaxor-type relaxation process can appear on cooling below $T_{g}$. It is notable that changes of temperature influence mainly the activation energy whereas compressing changes density, free volume and intermolecular distances. This explains why the Arrhenius process for the $\tau(T)$ behaviour is linked to the SA/SB process for the $\tau(P)$ evolution. All these can indicate a new possibility for modelling the dielectric properties of polymer-micro/nanoparticles composites, as well as for the insight into the still puzzling glass transition properties. For higher pressures, when approaching the GPa domain, the "metamaterial" behaviour emerges. It starts for BST/PVDF (10\% BST) already for $P \sim 0.6 \mathrm{GPa}$ and for $\mathrm{BST} / \mathrm{PVDF}(50 \% \mathrm{BST})$ for $P \sim 0.9 \mathrm{GPa}$. For the first composite, it manifests in the unusual, frequencycontrolled, spectrum associated also with unusual negative values of electric capacitance.

Such phenomenon was predicted decades ago and only recently directly evidenced experimentally in a thin, epitaxial ferroelectric film. It is worth recalling that the negative pressures phenomenon is considered as "the New Hope" for the low-energy nanoelectronics in the 21st cen- 
tury $[21,40-45]$. Generally, the appearance of the negative capacitance is expected for ferroelectric materials and considered as the consequence of the energy stored from the phase transition and the excess of charges appeared within the ferroelectric material [21,40-45]. In the opinion of the authors in PVDF/BST composites, particularly with the large enough amount of highly compressible polymer, extra-charges can be induced by the high-pressurerelated isotropic "push" on borders between PVDF polymer and BST particles.

The future extension of the given research can be the so-called thermodynamic scaling insight, both for PVDF and its composites. Basing on $\tau(T)$ for different isobars and $\tau(P)$ for different isotherms matched with $P-V-T$ thermodynamics data, the analysis of $\tau v s ., T V^{\gamma}$ behaviour offers also data on the intermolecular interactions. A nice resume of the most recent efforts in this fields, showing also new possibilities due to the simultaneous consideration of both the primary relaxation time and DCconductivity is given in ref. [46].

This picture can be supported by the well-known piezoelectricity of PVDF. It is notable that fig. 5 shows probably the first direct, stable and long-lived evidence of the negative capacitance phenomenon in the bulk material. Summarizing, this report shows different possibilities of inducing innovative and unusual metamaterial features in BST/PVDF microcomposites, achievable under still relatively moderate pressures.

The authors from IHPP PAS Poland (SS, SJR, EP) were supported by the NCN OPUS grant (ref. 2016/21/B/ST3/02203, headed by Aleksandra Drozd-Rzoska). Chinese coauthors were supported by the National Natural Science Foundation of China (No. 51672219), the International Cooperation Foundation of Shanaxi Province, China (No. 2017KW-025).

\section{Author contribution statement}

KZ and FG prepared the samples, made the structural and thermodynamic characterization and the temperature scans of dielectric permittivity from the melting temperature to the room temperature; this is also associated with writing-related sections in the report. SS, EP and AKS carried out BDS wide temperature range measurements in BST/PVDF composites. Data analysis was carried out by SS AD-R and SJR Key issues of the paper were formulated and written by ADR, SJR and SS, by discussing the impact with MS.

Publisher's Note The EPJ Publishers remain neutral with regard to jurisdictional claims in published maps and institutional affiliations.

Open Access This is an open access article distributed under the terms of the Creative Commons Attribution License (http://creativecommons.org/licenses/by/4.0), which permits unrestricted use, distribution, and reproduction in any medium, provided the original work is properly cited.

\section{References}

1. K.L. Ngai, Relaxation and Diffusion in Complex Systems (Springer, Berlin, 2011).

2. J.R. Fried, Polymers Science and Technology (Prentice Hall, Boston, 2014).

3. J.M.G. Cowie, V. Arrighi, Polymers: Chemistry and Physics of Modern Materials (CRC, Boca Raton, 2007).

4. S. Starzonek, S.J. Rzoska, A. Drozd-Rzoska, S. Pawlus, J.-C. Martinez-Garcia, L. Kistersky, Soft Matter 11, 5554 (2015).

5. F. Kremer, A. Schoenhalls Broad Band Dielectric Spectroscopy (Springer, Berlin, 2000).

6. S.J. Rzoska, A. Drozd-Rzoska, V. Mazur, Metastable Systems under Pressure (Springer, Berlin, 2010).

7. P.K. Mallick, Processing of Polymer Matrix Composites (CRC Press, Boca Raton, 2018).

8. S. Thomas, K. Joseph, S.K. Malhotra, K. Goda, M.S. Sreekala, Polymer Composites (Wiley-VCH, Berlin, 2010).

9. T. Hanemann, D.V. Szabó, Materials 3, 3468 (2010).

10. E. Fukada, T. Furukawa, Ultrasonics 19, 31 (1981).

11. K. Tashiro, K. Takano, M. Kobayashi, Y. Chatani, H. Tadakoro, Polymer 24, 199 (1983).

12. A.J. Lovinger, D.D. Davis, R.E. Cais, J. Kometani, Macromolecules 19, 1491 (1986).

13. J. Kaszyńska, B. Hilczer, P. Biskupski, Polym. Bull. 68, 1121 (2012).

14. V. Sencadas, S. Lanceros-Mendez, R. Sabater-Serra, A.A. Balado, J.L. Gomez Ribelles, Eur. Phys. J. E 35, 41 (2012).

15. E. Ozkazanc, H.Y. Guney, T. Oskay, E. Tarcan, Appl. Polym. Sci. 109, 3878 (2008).

16. E.N. Bunting, G.R. Shelton, A.S. Creamer, J. Res. Natl. Bur. Stand. 38, 337 (1947).

17. A. Kukreti, A. Kumar, U.C. Naithani, Ind. J. Pure. Appl. Phys. 49, 126 (2011).

18. J. Li, D. Jin, L. Zhou, J. Cheng, Mater. Lett. 76, 1 (2012).

19. S. Starzonek, K. Zhang, A. Drozd-Rzoska, S.J. Rzoska, E. Pawlikowska, M. Szafran, F. Gao, Polivinylidene difluoride-based composite: unique glassy and pretransitional behaviour, to be published in Eur. Phys. J. B (2019).

20. L.M. Garten, M. Burch, A.S. Gupta, R. Haislmaier, V. Gopalan, E.C. Dickey, S. Trolier McKinstry, J. Am. Ceram. Soc. 99, 1645 (2016).

21. A.M. Ionescu, Nat. Nanotechnol. 13, 7 (2018).

22. S.J. Rzoska, Front. Mater. Glass Sci. 4, 33 (2017).

23. P. Atkins, J. De Paula, Physical Chemistry for the Life Sciences (W. H. Freeman and Co., NY, 2018).

24. A. Drozd-Rzoska, S.J. Rzoska, Phys. Rev. E 65, 041701 (2002).

25. A. Drozd-Rzoska, S.J. Rzoska, Phys. Rev. E 73, 041502 (2006).

26. A. Drozd-Rzoska, S.J. Rzoska, S. Pawlus, Ll. J. Tamarit, Phys. Rev. B 73, 224205 (2006).

27. J.C. Martinez-Garcia, S.J. Rzoska, A. Drozd-Rzoska, J. Martinez-Garcia, Nat. Commun. 4, 1823 (2013).

28. C. Barus, Am. J. Sci. 45, 87 (1893).

29. H. Vogel, Phys. Z. 22, 645 (1921).

30. G.S. Fulcher, J. Am. Ceram. Soc. 8, 339 (1925).

31. G. Tammann, J. Soc. Glass Technol. 9, 166 (1925).

32. R. Boehmer, K.L. Ngai, C.A. Angell, J.D. Plazek, J. Chem. Phys. 99, 4201 (1993).

33. M. Paluch, S.J. Rzoska, J. Zioło, J. Phys.: Condens. Matt. 10, 4131 (1998). 
34. G.P. Johari, E.P. Whalley, Faraday Symp. Chem. Soc. 6, 23 (1972).

35. M. Paluch, J. Zioło, S.J. Rzoska, Phys. Rev. E 56, 5764 (1997).

36. C.A. Angell, Strong and fragile liquids, in Relaxations in Complex Systems, edited by K.L. Ngai, G.B. Wright (National Technical Information Service, U.S. Dept. of Commerce, Springfield, 1985).

37. G. Floudas, M. Paluch, A. Grzybowski, K. Ngai, Molecular Dynamics of Glass-Forming Systems: Effects of Pressure (Springer, Berlin, 2011).

38. A. Drozd-Rzoska, S.J. Rzoska, C.M. Roland, J. Phys.: Condens. Matt. 20, 244103 (2008).

39. S.J. Rzoska, A. Drozd-Rzoska, S. Starzonek, Nonlinear Dielectric Effect in Critical Liquids, in Nonlinear Dielectric Spectroscopy, Springer Ser. Adv. Dielectr., edited by Ranko Richert (Springer, Berlin, 2018).
40. G. Catalan, D. Jimenez, A. Gruvermanm, Nat. Mater. 14, 137 (2015).

41. A.I. Khan, K. Chatterjee, B. Wang, S. Drapcho, L. You, C. Serrao, S.R. Bakaul, R. Ramesh, S. Salahuddin, Nat. Mater. 14, 182 (2015).

42. P. Zubko, J.C. Wojdel, M. Hadjimichael, S. FernandezPena, A. Sene, I. Lukyanchuk, J.M. Triscone, J. Íñiguez, Nature 534, 524 (2016).

43. T. Sluka, P. Mokry, N. Setter, Appl. Phys. Lett. 111, 152902 (2017).

44. M. Si, Ch.-J. Su, C. Jiang, N.J. Conrad, H. Zhou, K.D. Maize, G. Qiu, Ch.-T. Wu, A. Shakouri, M.A. Alam, P.D. Ye, Nat. Nanotechnol. 13, 24 (2018).

45. A.K. Saha, S. Datt, S.K. Gupta, J. Appl. Phys. 123, 105102 (2018).

46. E. Thoms, A. Grzybowski, S. Pawlus, M. Paluch, J. Phys. Chem. Lett. 9, 1783 (2018). 